\title{
Architectural Distortion Detection in Mammogram using Contourlet Transform and Texture Features
}

\author{
S.Anand \\ Assistant Professor \\ Mepco Schlenk Engineering College \\ Sivakasi, Tamilnadu, India
}

\author{
R.Aynesh Vijaya Rathna \\ PG Student \\ Mepco Schlenk Engineering College \\ Sivakasi, Tamilnadu, India
}

\begin{abstract}
Breast Cancer is one of the most affecting diseases in the world. Architectural Distortion is one of the indications of breast cancer. It is an abnormal arrangement of tissue strands of the breast, often a radial or perhaps a somewhat random pattern, but without any associated mass as the apparent cause of this distortion. This project used Contourlet Transform based method to detect the location of the architectural distortion in mammograms. The dominant angle is detected in this Contourlet decomposition which comprises of Laplacian Pyramidal Filter bank and Steerable Gaussian Filter. The textural features will not show any deviation in the distorted area. Here the methods proposed will assist the Radiologist to identify the unidentified potential sites of distortion. Therefore the main aim of this work becomes the reduction of the region of interests. In this project 39 cases were testes in which 19 are Architectural Distorted image from the MIAS database and 17 Normal images. The proposed algorithm detected 1502 regions of interest (ROIs) with 247 True Positives and 1255 False Positives. In this paper 26 textural features were considered and result is obtained as 0.77 with an artificial neural network. The proposed methods have significant result in detecting the architectural distortion in mammograms of interval cancer cases.
\end{abstract}

\section{Keywords}

Breast Cancer, Contourlet Transform, Texture features.

\section{INTRODUCTION}

Breast cancer is a type of cancer originating from breast tissue, most commonly from the inner lining of milk ducts or the lobules that supply the ducts with milk. In India the breast cancer contributes $25 \%$ to $33 \%$ in urban areas [9]. In worldwide the breast cancer affects more women. Breast cancer is more than 100 times more common in women than in men, although men tend to have poorer outcomes due to delays in diagnosis. The breast cancer survival rate can be increased, if it is detected early. So the detection of the disease becomes imperative. The cancer affects the people by smoking, drinking, unbalanced diet etc. Researchers have proposed many techniques to detect this architectural distortion. Rangaraj M. Rangayyan et al. [24] proposed angular deviation of the oriented structures using a measure of coherence, to detect sites of architectural distortion. They tested 106 prior mammograms of 56 interval-cancer cases and 52 mammograms of 13 normal cases, and obtained the result with the sensitivity of $80 \%$ at 10.3 false positives/ image. Sujoy Kumar Biswas et al. [26] used the 19 Architectural
Distortion and 21 normal images (M1 dataset) and followed the leave-one-full mammogram out classification scheme. He recorded 3.6 false positives per image at $81.3 \%$ sensitivity with a ROI radius of $5 \mathrm{~mm}$ (minimum radius of $\mathrm{AD}$ region in MIAS) against 4.1 (at $80 \%$ sensitivity) and 7.8 (at sensitivity $84 \%$ ) false positives per image.

Shantanu Banik et al. [25] detected the site using the Gabor filters and phase portrait analysis. They automatically obtained 4224 ROIs from 106 prior mammograms of 56 interval-cancer cases, including 301 true-positive ROIs related to architectural distortion, and from 52 mammograms of 13 normal cases. Nemoto et al. [21] computed radiography (CR) digital mammograms, each with a single area of radiating spiculation of architectural distortion. They proposed the likelihood of spiculation and a modified point convergence index weighted by the likelihood to enhance architectural distortion. The result is with the sensitivity of $80.0 \%$ at 0.80 FP/image.

Guo et al [8] used 19 ROIs with architectural distortion and 41 with normal breast parenchyma, manually selected from the MIAS database. They applied lacunarity and five methods to estimate the Fractal Dimension with an SVM and differentiated the masses and architectural distortion from normal parenchyma and got the AUC of $0.875 \pm 0.055$.

Matsubara et al. [19] tried 280 mammograms in which 121 abnormal mammograms with architectural distortion and 159 normal images from the DDSM. He proposed the method by finding the thin mammary glands and got results of sensitivity of $75 \%$ at $2.9 \mathrm{FP} / \mathrm{image}$

Nakayama et al. [20] make use of 80 mammograms with architectural distortion. They detected the sites with the multiresolution analysis by decomposing the original image into several subimages at three scales by a novel filter bank based on wavelets and the Hessian matrix and got the sensitivity of $71.3 \%$ (57 out of 80 images) at 3.01 FP/image.

Ayres and Rangayyan [2] improved the result by using a bank of Gabor filters and constrained linear phase portrait modelling and obtained with the sensitivity of $84 \%$ at 4.5 FP/image. Eltonsy et al. [7] took 80 images in which 13 masses, 38 masses accompanied by architectural distortion, and 29 with only architectural distortion and implemented the method for locating points surrounded by concentric layers of image activity. The overall sensitivity of $91.3 \%$ with 9.1 $\mathrm{FP} /$ image is obtained. 
Hara et al. [10] used dynamic range compression as a preprocessing step before extracting the mammary gland by a combination of mean curvature and a shape index; a sensitivity of $70 \%$ was achieved at $2 \mathrm{FP} /$ image. Sampat et al. $[27,28]$ applied a linear filter to the Radon transform of the given image for the enhancement of spicules, the enhanced image was filtered with radial spiculation filters to detect spiculated masses and architectural distortion marked by converging lines or spiculation. He used a set of 45 images with spiculated masses and another set of 45 images with architectural distortion, he achieved was $91 \%$ at 12 FP/image and $80 \%$ at $14 \mathrm{FP} /$ image, respectively.

Özekes et al. [22] analysed the cases of architectural distortion from the mini-MIAS database. They proposed the method by computing the several distance thresholds to detect the architectural distortion and attained the accuracy of $89.02 \%$. Ayres and Rangayyan [1] yet again took 106 ROIs(Region of Interests) in which 17 cases of architectural distortion, 45 normals, two ROIs with malignant calcifications, and 44 masses. They characterized subtle changes and got the result of sensitivity $76.5 \%$ and specificity $76.4 \%$.

Tourassi et al. [30] worked with 112 ROIs with architectural distortion and 1,388 normal ROIs. They estimated Fractal Dimension with the circular average power spectrum technique and got the Area under the curve 0.89. Ichikawa et al. [12] attempted 94 mammograms with architectural distortion. They found the concentration index of linear structures obtained by the mean curvature of the image and the result as the sensitivity of $68 \%$ with $3.4 \mathrm{FP} / \mathrm{image}$.

Matsubara et al. [18] used 55 mammograms with architectural distortion in that 17 with focal retraction, 38 with architectural distortion within the fibro glandular disk. They proposed the method of morphological image processing with a concentration index and obtained the result as around the skin line sensitivity of $94 \%$ with $2.3 \mathrm{FP} /$ image; within the mammary gland: sensitivity of $84 \%$ with $2.4 \mathrm{FP} /$ image as the result.

Karssemeijer and Te Brake [14] took 31 normal cases and 19 cases with stellate lesions from the MIAS database. A multiscale-based method for the detection of stellate distortion including spiculating masses and architectural distortion using the output of three-directional, second order, Gaussian derivative operators is implemented. They did outcome with a sensitivity of about $90 \%$ at one FP per image.

This project presents a system developed for the detection of Architectural distortion (AD) in the screening program. This paper used the Contourlet Transform (CT) that comprised of Laplacian Pyramidal Filter and Steerable filter, the Dominant image which is generated by the different angles of Steerable Gaussian filter and the Textural analysis with Artificial Neural Network (ANN) used for the area detection. The image enhancement is done by using this Contourlet transform and orientations of the mammogram were enhanced by the proposed Dominant angle image that is found from the Steerable filter. The texture features along with the Neural Network scheme reduces the ROI towards the detection of Architectural Distorted area.

In this Chapter the overview of the project is given with the literature survey. The literature survey clearly depicts the various authors, their proposed methods and their results. The second chapter describes the proposed method towards the detection of the Architectural Distortion area. It also includes the steps in detecting the defect area. They are the preprocessing technique (Morphological Filter), Image enhancement by the Contourlet transform with the Dominant angle methods. Then this chapter briefly describes the Textural analysis of Law's Texture features, Haralick's feature, Roughness measure and Fractal Dimension features. It also gives the basics of the Artificial Neural Network as its use for the classification. The third chapter presents the result with its performance analysis.

\section{PROPOSED METHOD}

\subsection{Introduction}

The proposed method towards the detection of Architectural distortion is discussed in detail in this chapter. The following flow chart (Figure 2.1) shows the automatic detection of the location of the Architectural Distortion area. Hence our aim is to find the area of Architectural distortion, this paper used the textural analysis to classify the Normal image with the Architectural Distorted image and for later case this paper used the Artificial Neural Network.

\subsubsection{Flow Chart with Description}

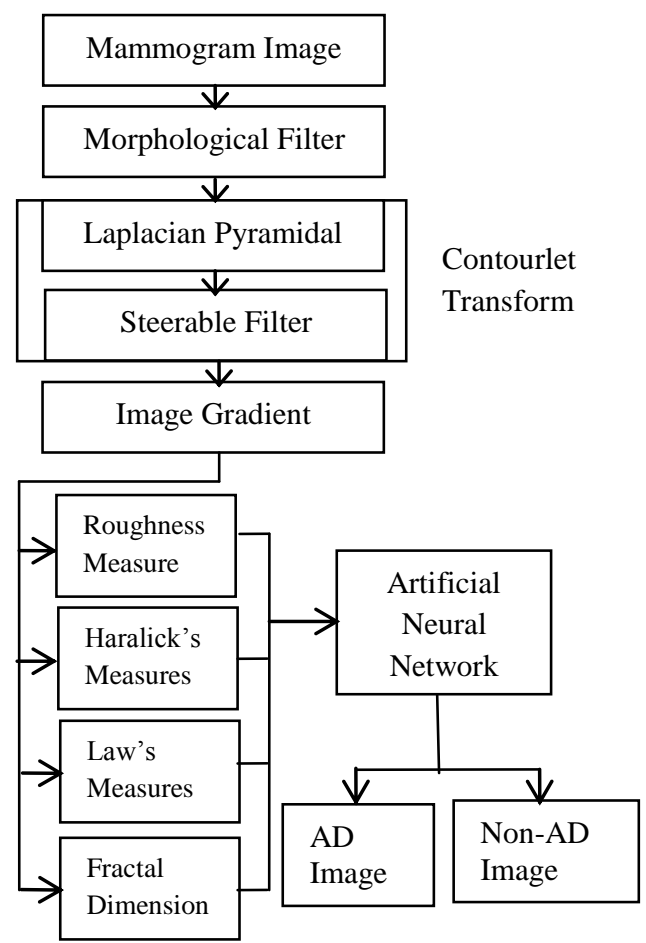

Figure 2.1 Flow Chart of Detection of Architecture Distorted Area.

The mammogram image is pre-processed using the morphological filter and this filtered output is given to the Contourlet Transform (comprised of Laplacian Pyramidal Filter and Steerable Filter) to enhance it. To enhance the image with more orientation the Steerable filter is applied at different angles as a directional filter in the Contourlet transform. For each angle a directional image and magnitude image is computed by using the two point equations. The dominant angle image is obtained by comparing and obtaining the maximum direction and corresponding magnitude image. The obtained dominant magnitude image is the enhanced image of our proposed image. The texture features such as Roughness, Law's Texture analysis and Haralick's Texture features and Fractal Dimension are found from the enhanced images that contain both Architectural Distorted image and normal images. An Artificial Neural Network is trained with the 26 computed features. In testing a test image of size 1024 
x 1024 is split as $100 \times 100$ and each image is pre processed and the above procedure is followed till computing all the 26 feature values. The computed values are again fed to the Artificial Neural Network. The ANN model is simulated with the previously computed training set of feature values to obtain the data under the curve. This model is simulated to find the ROC and this value is compared with the experimental value and the classification is obtained.

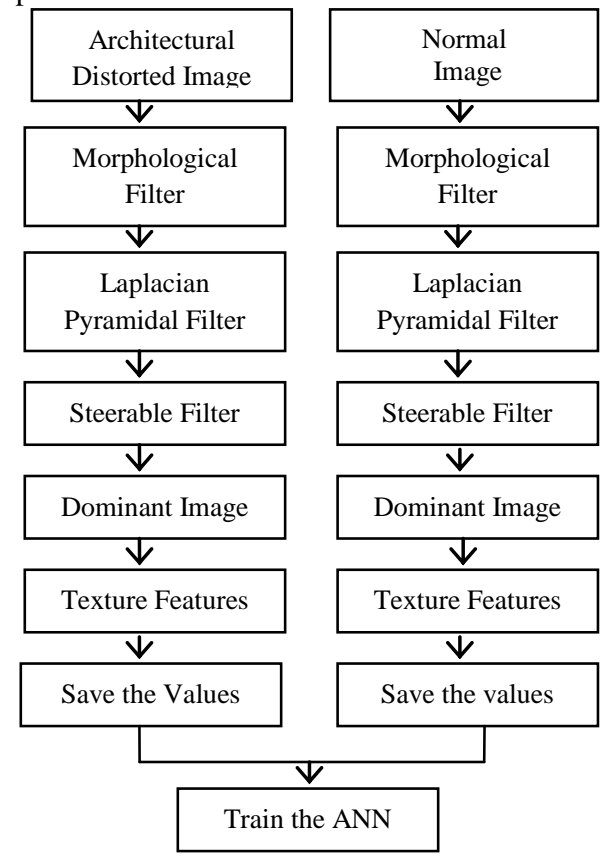

Figure 2.2 Flow Chart of Training the ANN Network

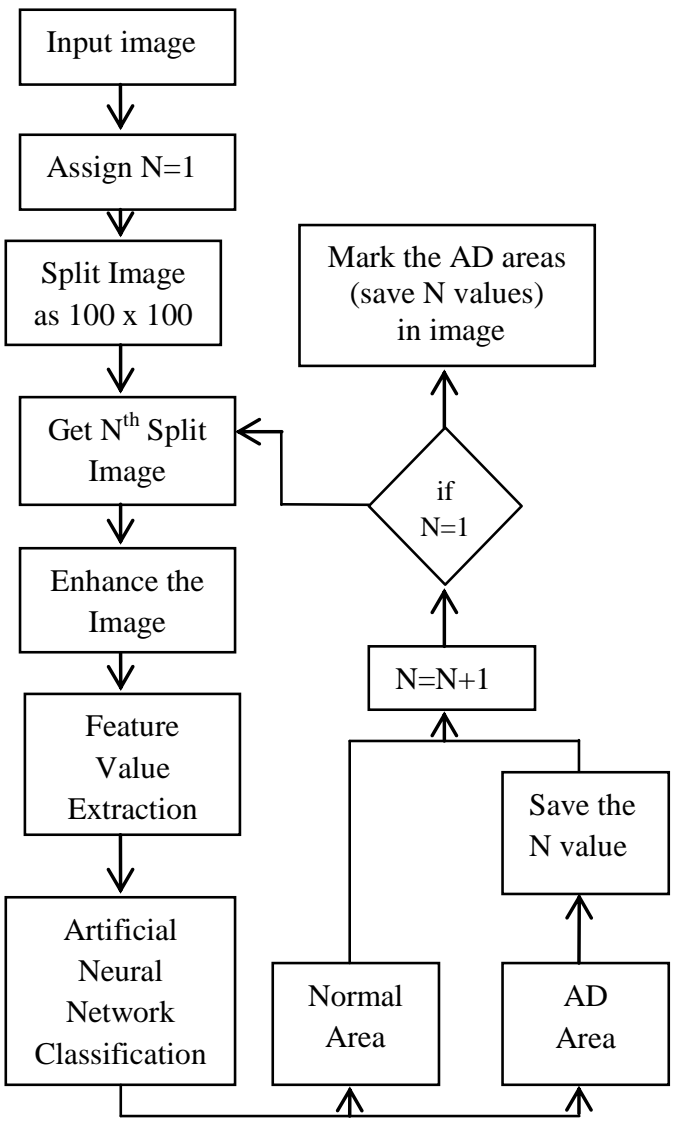

Figure 2.3 Flow Chart of Detection of ROI
The results of the detection of Architectural Distortion are arrived by the shown two Flow Charts 2.2 and 2.3. The Flow chart 2.2 is used to train the feature values and the Flow Chart 2.3 is used to detect the ROIs of the potential sites of the Architectural Distortion. A training set of images of size 100 $\mathrm{x} 100$ is selected from the Architectural distortion images and normal images for training the Artificial Neural Network. The split image is pre processed with the morphological filter and Contourlet Transform. The Dominant angle is obtained from the different angle output of the Steerable filter.

The Textural features are computed for each resultant image and this is fed to the Artificial Neural Network for training and the data are stored for feature comparison. The detection of ROI is performed as shown in the Flow chart 2.3. The input image of size 1024 x 1024 is split to the size of $100 \times 100$ and the hypothesis is assumed here is that the Architectural Distortion site is present in the area within the input image.

The steps till finding the textural feature are same as the training algorithm for testing algorithm except the classification by the ANN model. The ANN model is simulated between the obtained values of textural features both from the training and testing and if the resultant value is below 0.75 then the split image is considered as the normal image and if it is above the prescribed value then it is classified as Architectural Distorted image. The location of the image is identified and a rectangle is drawn to locate the Architectural Distortion in the original image.

\subsection{MORPHOLOGICAL FILTER}

Morphological filter is a technique used for the analysis and processing of geometrical structures. This filter is based on set theory, lattice theory, topology, and random functions. In digital images processing the Morphology [33] is most commonly applied.

\subsubsection{Top-Hat Transform}

The Top-Hat transform extracts small elements and details from given images. There are two types of top-hat transform. First one is white top-hat transform, defined as the difference between the input image and its opening by some structuring element. The second is the black top-hat transform is defined dually as the difference between the closing and the input image. Top-hat transforms are used for various image processing tasks, such as feature extraction, background equalization, image enhancement, and others.

\subsubsection{Mathematical Definitions}

Let $f: E \rightarrow R$ be a grayscale image, mapping points from a Euclideanspace or discrete grid $\mathrm{E}$ into the real line.

Let $\mathrm{b}(\mathrm{x})$ is a grayscale structuring element.

Then, the white top-hat transform of $f$ is given by the equation 2.1 ,

$$
T_{\omega}(f)=f-f_{\mathrm{o}} b
$$

$$
\text { where o denotes the opening operation. }
$$

The black top-hat transform of $f$ is given by the equation 2.2 ,

$$
T_{b}(f)=f \bullet b-f
$$$$
\text { where } \bullet \text { is the closing operation. }
$$

\subsection{CONTOURLET TRANSFORM}

The Contourlet Transform [6] is a multiscale, multi resolution filter that comprised of Pyramidal filter and Directional filter. The Proposed algorithm used Laplacian Pyramidal filter as Pyramidal filter and Steerable filter as directional filter. The Contourlet Transform enhances the image with its property of decomposition and reconstruction. 


\subsubsection{Laplacian Pyramid Filter}

The Laplacian Filter highlights regions of rapid intensity changes. The Laplacian Filters smooth the input image using a Gaussian smoothing filter in order to reduce its sensitivity to noise. The Laplacian Filter decomposes the images into information at multiple scales. This filter extracts features of interest and to attenuate noise that present in the image. The applications of this filter can be image enhancement, restoration and image analysis. The efficient image coding for the image modelling is achieved by the property of the redundancy reduction of this filter. The Laplacian filter consists of Low pass filter and High pass filter. The decomposition is based on the difference between the two filters. The image is recursively decomposed into low-pass and high-pass bands. In each decomposition level the LP [3] creates a down sampled low-pass version of the original image and a band-pass image.

\subsubsection{Steerable Gaussian Filter}

The Steerable Pyramid [32] is linear multi-scale, multiorientation image decomposition filter. It consists of rotationinvariant linear operators such as Gaussians and Hibbert transforms. This filter is used for many applications such as orientation and phase analysis, angularly adaptive filtering, edge detection and shape from shading. The Steerable Filter is used to form a multiscale, self-inverting pyramidal decomposition. The transform coefficients are obtained by reconstruction of the results acquired by the filters applied on each time. The result is a low-passed version of the original image. Orientation analysis in all scales is controlled by this Steerable filter. The Steering process is the synthesis of orientation filter at any given angle.

\subsection{IMAGE GRADIENT}

An image gradient [23] is a directional change in the intensity or color in an image. Image gradients may be used to extract information from images.

\subsubsection{Gradient Computation}

Mathematically, the gradient of a two-variable function at each image point is a Two Dimensional vector with the components given by the derivatives in the horizontal and vertical directions. At each image point, the gradient vector points in the direction of largest possible intensity increase, and the length of the gradient vector corresponds to the rate of change in that direction.

The gradient of an image measures how it is changing. It provides two quantities of information. The magnitude of the gradient tells us how quickly the image is changing, while the direction of the gradient tells us the direction in which the image is changing most rapidly.

The gradient of a function of two variables, $F(x, y)$, is defined in the equation 2.3 ,

$$
\nabla \mathrm{F}=(\partial \mathrm{F} / \partial \mathrm{x}) \mathrm{i}+(\partial \mathrm{F} / \partial \mathrm{y}) \mathrm{j}
$$

and can be thought of as a collection of vectors pointing in the direction of increasing values of F. In MATLAB software, numerical gradients (differences) can be computed for functions with any number of variables. For a function of $\mathrm{N}$ variables, $\mathrm{F}(\mathrm{x}, \mathrm{y}, \mathrm{z} \ldots)$ the values are given in the equation 2.4,

$$
\nabla f=(\partial \mathrm{F} / \partial \mathrm{x}) \mathrm{i}+(\partial \mathrm{F} / \partial \mathrm{y}) \mathrm{j}+(\partial \mathrm{F} / \partial \mathrm{y}) \mathrm{k}
$$

The Gradient of a 2D function, $\mathrm{f}(\mathrm{x}, \mathrm{y})$, is defined as the vector and it is given in the equation 2.5 ,

$$
\nabla f=\operatorname{grad}(f)=\left[\begin{array}{c}
g_{x} \\
g_{y}
\end{array}\right]=\left[\begin{array}{c}
\frac{\partial f}{\partial x} \\
\frac{\partial f}{\partial y}
\end{array}\right]
$$

whose magnitude of the image is given by the equation 2.6,

$$
\left.M(x, y)=\operatorname{mag}(\nabla f)=\sqrt{\left(g_{x}^{2}\right.}+g_{y}^{2}\right)
$$

And whose direction is given by the angle $\alpha$ and this is represented in the equation 2.7,

$$
\alpha(x, y)=\tan ^{-1}\left[\begin{array}{l}
g_{y} \\
g_{x}
\end{array}\right]
$$

where $\alpha$ is measured with respect to the axis and is an image of the same size as the original created by the array division of image $g_{y}$, by image $g_{x}$.

The direction of an edge at an arbitrary point $(\mathrm{x}, \mathrm{y})$ is orthogonal to direction, $\alpha(x, y)$, of the gradient vector at the point

\subsection{DOMINANT ANGLE DETECTION}

Dominant angle image provides an enhanced image with orientation by comparing the peak values. Dominant angle detection is derived from Magnitude and Phase images of different angle.

\subsubsection{Estimation of Dominant Angle}

The dominant angle detection needs Gradient of images. The Gradient image consists of Magnitude image and Phase image. The Gradient images need to be calculated for many angles. The amplitude of magnitude images were compared at each pixel and the maximum amplitude are detected. The angle at which the maximum amplitude occur the corresponding value of the Phase image is stored as the dominant image [17].

The dominant angle and Dominant Phase image were calculated by the following equations 2.8 and 2.9,

$M(x, y)=\max (G M(x, y))$ for $\theta=0^{0}$ to $180^{\circ}$

$$
P(x, y)=G P_{\theta}(x, y)
$$

where, to $180^{\circ}$

$\theta$ belongs to location of $\max (G M(x, y))$ for $\theta=0^{0}$
$M(x, y) \quad-$
$P(x, y)-$
Dominant Magnitude Image
$\operatorname{GM}(x, y)-$
Dominant Phase Image
$G P(x, y)-$
Gradient Magnitude Image
Gradient Phase Image

Analysis of the dominant orientation of texture patterns often provides important information regarding the object under consideration, and could be used in characterizing or deriving discriminated features for the object. On the other hand, analysis of random patterns associated with certain regions in an image could also help in generating useful features for the detection abnormalities. As a result, multiple methods are required to characterize the variety of texture found in mammograms.

\subsection{TEXTURE ANALYSIS}

An image containing several different regions will have distinct texture. The texture composed of texels, and statistics. To distinct the parts analysis of the texture of the image is needed.

In this paper there are four texture categories were analyzed. They are Roughness measure, Haralick's Features, law's Texture features and Fractal dimension features. These four features were briefly discussed below.

\subsubsection{Roughness Measure}

Roughness features are computed in different directions in an effort to simulate the human visual system, which perceives roughness in different directions. The visual effect of directional roughness depends highly on the relative textural energy in different directions. The roughness features 
computed in a direction with the percentage of textural energy in the same direction.

Roughness is a measure of the texture of a surface. It is quantified by the vertical deviations of a real surface from its ideal form [5]. If these deviations are large, the surface is rough; if they are small the surface is smooth. Roughness is typically considered to be the high frequency, short wavelength component of a measured surface.

$$
\left\langle\max _{u \in(x-\epsilon, x+\varepsilon)}\left|W_{i} T_{f}^{\theta}(u, v, s)\right|\right\rangle_{N \times N} \sim \varepsilon^{R_{s t}^{\theta}}
$$

The equation 2.10 gives the roughness measure. where \langle\rangle indicated spatial arithmetic average in an $\mathrm{N} \times \mathrm{N}$ window, $\mathrm{i}=$ 1,2 ( first and second derivative, respectively) and $\mathrm{W}$ is the wavelet transform. The directional features are computed as the slope of the line that best fits. The above equation gives the roughness measure of any two dimensional image.

\subsubsection{Haralick's Features}

Haralick developed the Gray-level Co-Occurrence matrices that produce intensity change histograms as a function of distance and direction. By far the most popular texture description method is Gray level Co-occurrence matrices due to its simplicity [11].

The co-occurrence matrix is an estimate of the second order joint probability, the probability calculated by going from gray level $\mathrm{i}$ to gray level $\mathrm{j}$, given the distance $\mathrm{d}$ between two pixels along a given direction $\theta$.

The formulas to find the Haralick's Texture features were listed in the following equations,

The Contrast is given in equation 2.11,

Contrast $=\sum_{i, j}|i-j|^{2} p(i . j)$

The Energy is represented in equation 2.12 ,

Energy $=\sum_{i . j} p(i, j)^{2}$

The Dissimilarity is given by the equation 2.13

Dissimilarity $=\sum_{i, j=0}^{N-1} P_{i . j}^{i-j}$

The Cluster Prominence is represented by the equation 2.14,

Cluster Prominence $=\sum_{\substack{i=0 \\ j=0}}^{G-1}\left\{i+j-\mu_{x}-\mu_{y}\right\}^{4} p(i, j)$

The Cluster Shade is given in the equation 2.15,

Cluster shade $=\sum_{i=0}^{G-1} \sum_{j=0}^{G-1}\left\{i+j-\mu_{x}-\mu_{y}\right\}^{3} p(i, j)$

The Entropy is given by the equation 2.16 ,

Entropy $=\sum_{i, j=0}^{N-1} P_{i, j}\left(-\ln P_{i, j}\right)$

The Homogeneity is represented by the equation 2.17,

Homogeneity $=\sum_{i . j=0}^{N-1} \frac{P_{i, j}}{1+(i-j)^{2}}$

The Variance can be calculated by the given equation 2.18,

Variance $=\sigma_{i}^{2}=\sum_{i, j=0}^{N-1} P_{i, j}\left(i-\mu_{i}\right)^{2}$

\subsubsection{Laws Texture Energy Measures}

Laws' texture energy measures are based on convolution kernels that emphasize specific structural patterns, and could be used to generate useful features related to the intersecting tissue structures, spiculations, and node-like patterns of architectural distortion. Laws [16] defined several 1D and 2D convolution kernels to classify each pixel in an image based on measures of local "texture energy".

The texture energy features represent the amounts of variation within a sliding window applied to several filtered versions of the given image. The basic operators in Laws' method are:

$$
\begin{aligned}
& \text { L3 }=[1,2,1], \\
& \text { E3 }=[-1,0,1], \text { and } \\
& \text { S3 }=[-1,2,-1],
\end{aligned}
$$

where L3,E3, and S3 perform center-weighted averaging, symmetric first differencing (edge detection), and second differencing (spot detection), respectively. Nine $3 \times 3$ masks can be generated by multiplying the transposes of the three operators with their direct versions. For example, the result of L3T E3 gives one of the $3 \times 3$ Sobel masks as

$$
\left[\begin{array}{lll}
-1 & 0 & 1 \\
-2 & 0 & 2 \\
-1 & 0 & 1
\end{array}\right]
$$

There are nine convolution masks are available in this Law's Texture energy measures. They are L5E5/E5L5 L5S5/S5L5, L5R5/R5L5 E5E5, E5S5/S5E5 E5R5/R5E5, S5S5 S5R5/R5S5 and R5R5. Each measure can be multiplied with transposed features to get the two dimensional feature. The Texture measures were listed below with their masks.

$$
\begin{aligned}
\text { L5 (Level) } & =\left[\begin{array}{llll}
1 & 4 & 6 & 4
\end{array}\right] \\
\text { E5 (edge) } & =\left[\begin{array}{lllll}
-1 & -2 & 0 & 2 & 1
\end{array}\right] \\
\text { S5 (Spot) } & =\left[\begin{array}{lllll}
-1 & 0 & 2 & 0 & -1
\end{array}\right] \\
\text { R5 (Ripple) } & =\left[\begin{array}{lllll}
1 & -4 & 6 & -4 & 1
\end{array}\right]
\end{aligned}
$$

The first step to find the Law's Texture measures is the local average from each pixel is subtracted. Then the $165 \times 5$ masks applied to the preprocessed image and 16 filtered images are generated. The texture energy map $\mathrm{E}_{\mathrm{k}}$ for filter $\mathrm{k}$ is computed by the following equation 2.19 ,

$$
\mathrm{E}_{\mathrm{k}}[\mathrm{r}, \mathrm{c}]=\sum_{i=r=7}^{r+7} \sum_{j=c=7}^{c+7} F k[i, j]
$$

where $F_{k}[i, j]$ is the result of filtering with the $k$-th mask at pixel[i,j]. Nine 1D operators of length five pixels can be generated by convolving L3, E3, and S3 operators in different combinations; for example

$$
\begin{aligned}
& \mathrm{L} 5=\mathrm{L} 3 * \mathrm{~L} 3=[1,4,6,4,1], \\
& \mathrm{R} 5=\mathrm{S} 3 * \mathrm{~S} 3=[1,-4,6,-4,1], \\
& \mathrm{W} 5=-\mathrm{E} 3 * \mathrm{~S} 3=[-1,2,0,-2,1], \\
& \mathrm{E} 5=\mathrm{L} 3 * \mathrm{E} 3=[-1,-2,0,2,1], \\
& \mathrm{S} 5=-\mathrm{E} 3 * \mathrm{E} 3=[-1,0,2,0,-1],
\end{aligned}
$$

where $*$ represents $1 \mathrm{D}$ convolution. The operators L5, R5, W5, E5, and S5 can be used to perform the detection of features related to local average, ripples, waves, edges, and spots.

\subsubsection{Fractal Dimension (FD)}

The fractal property of self-similarity has been observed in many biomedical systems and images. Several studies have suggested that fractal analysis can be used to describe many complex natural phenomena by providing insight into the intrinsic characteristics of the system under investigation [13]. Consequently, fractal analysis has found various applications in medical signal and image processing and analysis.

\subsection{ARTIFICIAL NEURAL NETWORK}

An artificial neural network is used as a classifier in this present work. This artificial neural network is intelligent based computational simulation of a biological neural network. This model depicts the real life behaviour of neurons and the electrical messages they produce between input, processing by the brain and the final output from the brain [4].

In the present work, a multilayer neural network [15] with sigmoidal activation function is used. The overfitting and underfitting avoided by single neuron.

\section{RESULT}

In this project the detection of the Architectural distortion work is divided into two steps. First step is training the Artificial Neural Network with the selected training set of images of size $100 \times 100$ after the preprocessing, enhancement operation with the feature values. The training set of images of size $100 \times 100$ is selected randomly from the MIAS database. The split image is preprocessed with the 
morphological filter and Contourlet Transform is performed. The Dominant angle is obtained from the different angle output of the Steerable filter. The Textural features are computed for each resultant image and this is fed to the Artificial Neural Network for training and the data are stored for feature comparison. The detection of ROI is performed as shown in the Flow chart 2.3. The input image is split as $100 \mathrm{x}$ 100 and the hypothesis is assumed here is that the Architectural Distortion site is present in the area within it.

The steps till finding the textural feature is same as the training algorithm and the classification is done by simulating the ANN model between the obtained values of textural features both from the training and testing. If the resultant value is below 0.75 (this value is found by experimental by giving the Architectural Distortion and Normal Images) then the split image is considered as the normal image or if it is above the prescribed value then it is classified as Architectural Distorted image.

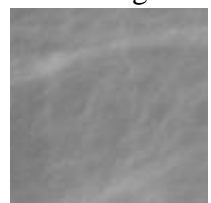

(a)

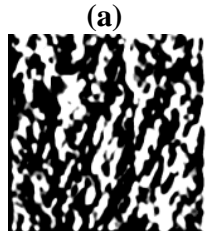

(d)

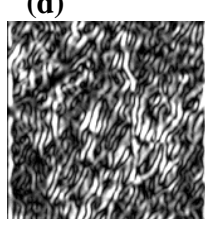

(g)

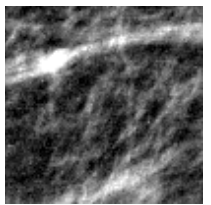

(b)

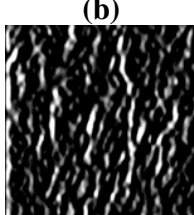

(e)

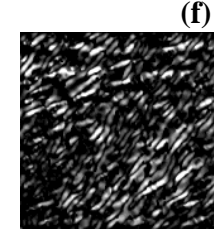

(h)
Figure 3.1 Detection of Dominant Image for mdb_125

(a) Input Image mdb_167.tif

(b) Morphological Filter Image

(c) Laplacian Pyramid Directional Filter

(d) Steerable Filter for $\mathbf{1 5}^{\circ}$

(e) Gradient Angle Image

(f) Gradient Magnitude Image

(g) Dominant Magnitude Image

(h) Dominant Angle Image

The location of the image is identified and a rectangle is drawn to locate the Architectural Distortion in the original image. The input image has shown in the Figure 3.1 (a). This input image is taken from the MIAS database [29] and the area of Architectural Distortion is split by concerning the ground truth given in the database. The Figure 3.1 (a) is $100 \mathrm{x}$ 100 split Architectural Distortion area from mdb_125.

A disk shaped opening filter was used for smoothing the edges. The resulting images clearly show the edges which separates the black and white regions. Therefore the ducts in the breast area are enhanced by this method and the resulting image will have the skin area as more black and the nerves and ducts to be brighter. The resulting image Figure 3.1 (b) shows the morphological output.

In the Laplacian Pyramidal filter, decomposition is based on difference-of-lowpass filters and the image is recursively decomposed into low-pass and high pass bands. The filter creates a high fidelity image. The output of this filter is an enhanced image with multi directional noise rejection filter. The results are shown in Figure 3.1 (c) for the input images mdb_125.

The Steerable filter is a single directional filter that is used to enhance the image with orientation. The result obtained after the application of this filter gives the better oriented output. The figure shows the enhanced image that gave the adaptive control and as well as orientation. The resultant images are shown in the Figure 3.1 (d) for the angle $15^{0}$. The steerable filter is applied after the application of the Laplacian Pyramidal filter.

Table 3.1 Textural Feature Extracted Values

\begin{tabular}{|l|c|c|}
\hline \multicolumn{1}{|c|}{ Feature Name } & $\begin{array}{c}\text { Architectural } \\
\text { Distorted } \\
\text { Image } \\
\text { (mdb_125) }\end{array}$ & $\begin{array}{c}\text { Normal } \\
\text { area image } \\
\text { test16.tif }\end{array}$ \\
\hline Law energy1 & $3.038 \mathrm{e}+13$ & $6.846 \mathrm{e}+12$ \\
\hline Law energy 2 & $2.142 \mathrm{e}+10$ & $4.941 \mathrm{e}+09$ \\
\hline FDF Average & $1.866 \mathrm{e}+00$ & $1.866 \mathrm{e}+00$ \\
\hline $\begin{array}{l}\text { FD Standard } \\
\text { Deviation }\end{array}$ & $6.008 \mathrm{e}-03$ & $1.656 \mathrm{e}-02$ \\
\hline FD Lacurinity & $1.054 \mathrm{e}-05$ & $9.445 \mathrm{e}-05$ \\
\hline Roughness Magnitude & $5.306 \mathrm{e}-01$ & $6.908 \mathrm{e}-01$ \\
\hline Roughness Angle & $2.191 \mathrm{e}-01$ & $3.126 \mathrm{e}-01$ \\
\hline Autocorrelation & $1.689 \mathrm{e}+04$ & $2.658 \mathrm{e}+04$ \\
\hline Contrast & $1.084 \mathrm{e}+04$ & $1.293 \mathrm{e}+04$ \\
\hline Correlation & $1.566 \mathrm{e}-02$ & $-1.058 \mathrm{e}-01$ \\
\hline Cluster Prominence & $2.942 \mathrm{e}+08$ & $2.848 \mathrm{e}+08$ \\
\hline Cluster Shade & $-1.603 \mathrm{e}+05$ & $5.321 \mathrm{e}+05$ \\
\hline Dissimilarity & $8.4552 \mathrm{e}+01$ & $9.134 \mathrm{e}+01$ \\
\hline Energy & $2.484 \mathrm{e}-05$ & $6.060 \mathrm{e}-05$ \\
\hline Entropy & $1.069 \mathrm{e}+01$ & $10.212+$ \\
\hline Homogeneity & $3.646 \mathrm{e}-03 \mathrm{i}$ & $4.866 \mathrm{e}-03 \mathrm{i}$ \\
\hline Maximum probability & $1.059 \mathrm{e}-04$ & $3.605 \mathrm{e}-02$ \\
\hline Variance & $2.140 \mathrm{e}+04$ & $4.085 \mathrm{e}-04$ \\
\hline Sum average & $2.593 \mathrm{e}+02$ & $2.896 \mathrm{e}+04$ \\
\hline Sum variance & $6.533 \mathrm{e}+04$ & $3.368 \mathrm{e}+02$ \\
\hline Sum entropy & $6.038 \mathrm{e}+00$ & $5.193 \mathrm{e}+05$ \\
\hline Difference variance & $1.084 \mathrm{e}+04$ & $1.293 \mathrm{e}+00$ \\
\hline Difference entropy & $5.351 \mathrm{e}+00$ & $5.413 \mathrm{e}+00$ \\
\hline $\begin{array}{l}\text { Information measure } \\
\text { of correlation }\end{array}$ & $-4.244 \mathrm{e}-02+$ & $-4.946 \mathrm{e}-02+$ \\
\hline $\begin{array}{l}\text { Inverse difference } \\
\text { normalized }\end{array}$ & $1.036 \mathrm{e}-03 \mathrm{i}$ & $8.888 \mathrm{e}-04 \mathrm{i}$ \\
\hline $\begin{array}{l}\text { Inverse difference } \\
\text { moment normalized }\end{array}$ & $6.644 \mathrm{e}-01$ & $6.640 \mathrm{e}-01$ \\
\hline Ordit & $8.695 \mathrm{e}-01$ & $8.625 \mathrm{e}-01$ \\
\hline
\end{tabular}

In order to get the dominant angle image two images needed as the Magnitude and the Angle image. This cannot be obtained from the Steerable filter. So from the output of the Steerable filter image the Gradient of images were found.

The Figure 3.1 (e) and 3.1 (f) are the Gradient Angle image and Gradient Magnitude image from the Steerable Filter output respectively. The magnitude images were found from the distance of two points and the angle is calculated by using the tangent of the point. The above mentioned images shown were for the angle of 15 degree.

Dominant angle image is found from the Magnitude image and Angle images of different angles from $15^{\circ}$ to $180^{\circ}$. At every 15 degrees total of 12 images were obtained from the steerable filter. The dominant angle of images were computed by comparing the angle images and finding the maximum angle value and the corresponding magnitude value is used to 
create a dominant magnitude image and angle image. The Figure 3.1 (g) and Figure 3.1 (h) gives the dominant magnitude image and dominant angle image for image mdb_125.

The texture features are tabulated in the Table 3.1. The values are listed for an architectural area of ROI (mdb_125) and for a Normal ROI (test16). The textural features were computed for the Roughness measure, Law's Texture Energy measures, Haralick's Feature and Fractal Dimension. In total 26 textural features were calculated and all the features were used to train the artificial neural Network and to test the Architectural Area also. Owing to the nature of these listed values have very little difference and intelligent techniques needed to classify these values.

The figure 3.2 shows the resultant image of our project that has the detected ROIs marked in the image. The resultant image has 15 ROIs in which there are 4 True Positive areas and 11 false positive areas marked.

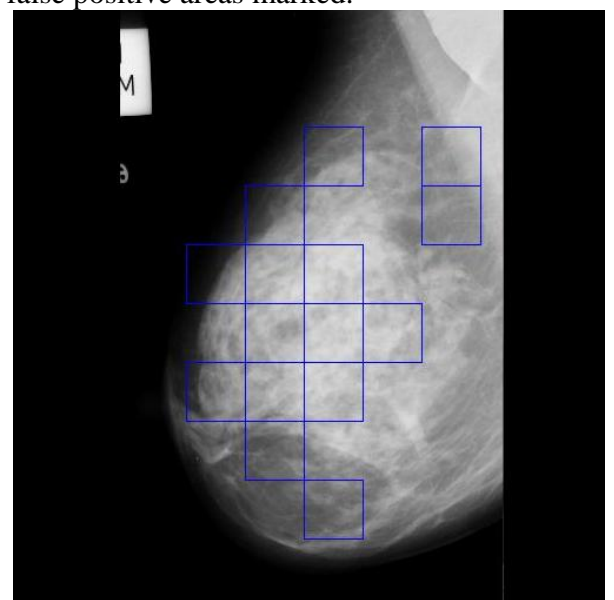

Figure 3.2Detection of ROI for mdb_125

In this project 39 cases were testes in which 19 are Architectural Distorted image from the MIAS database and 17 Normal images. The performance analysis of the images was listed in the Table 3.2. The average sensitivity of $64 \%$ is achieved through this analysis. Even though the results are convincing and this cannot be used for the clinical purposes and this need to be improved by the accurate model of the design and the using several constraints to it.

Table 3.2 Performance Analysis

\begin{tabular}{|l|c|}
\hline Total Number of Images Testing & 35 \\
\hline No. of Normal Images trained & 16 \\
\hline No. of Architectural Image & 19 \\
\hline No. of ROI created & 1502 \\
\hline True Positive & 247 \\
\hline False Positive & 1255 \\
\hline Sensitivity & $64 \%$ \\
\hline
\end{tabular}

\section{CONCLUSION}

The architectural distortion, breast cancer is automatically detected in this project. To detect the architectural distortion, modified Contourlet transform that comprised of Laplacian Pyramidal filter and Steerable Gaussian filter is used. With this finding the dominant angle of the mammogram enhanced the image with orientation. The Textural features were used to indicate the potential site of architectural distortion. Since these values have very narrow deviation in it, the intelligent classifier Artificial Neural Network also used in this project. The proposed algorithm detected 1502 regions of interest
(ROIs) with 247 True Positives and 1255 False Positives. The result obtained by this proposed algorithm is $64 \%$. The result can be improved by adding more number of textural features and feeding the training network with the selected features instead giving all the features. The proposed methods have significant in detecting architectural distortion in mammograms of interval cancer cases.

\section{REFERENCES}

[1] Ayres F. J. and Rangayyan R. M., "Characterization of architectural distortion in mammograms", IEEE Engineering in Medicine and Biology Magazine, Vol. 24, No. 1, pp. 59-67, 2005.

[2] Ayres F. J. and Rangayyan R. M., "Reduction of false positives in the detection of architectural distortion in mammograms by using a geometrically constrained phase portrait model", International Journal of Computer Assisted Radiology and Surgery, Vol. 1, pp. 361-369, 2007.

[3] Burt P. J. and Adelson E. H., "The Laplacian pyramid as a compact image code," IEEE Transactions on Communication, Vol. 31, No. 4, pp. 532-540, 1983.

[4] Chan H.P., Sahiner B., Wagner R. F. and Petrick N., "Classifier design for computer-aided diagnosis: Effects of finite sample size on the mean performance of classical and neural network classifiers", Medical Physics, Vol. 26, No.12, pp. 2654-2668, 1999.

[5] Dimitrios Charalampidis and Takis Kasparis, "WaveletBased Rotational Invariant Roughness Features for Texture Classification and Segmentation", IEEE Transactions On Image Processing, Vol. 11, No. 8, pp. 825-837, 2002.

[6] Do M. N. and Vetterli M., "The contourlet transform: An efficient directional multiresolution image representation”, IEEE Transactions on Image Processing, Vol. 14, No. 12, pp. 2091-2106, 2005.

[7] Eltonsy N., Tourassi G. and Elmaghraby A. "Investigating performance of morphology based CAD scheme in detecting architectural distortion in screening mammograms", International Congress and Exhibition on Computer Assisted Radiology and Surgery (CARS 2006), pp. 336-338, 2006

[8] Guo Q., Shao J. and Ruiz V., "Investigation of support vector machine for the detection of architectural distortion in mammographic images", Journal of Physics, Conference Series. 15, pp. 88-94, 2005.

[9] http://www.breastcancerindia.net/bc/statistics/trends.htm

[10] Hara T., Makita T., Matsubara T., Fujita H., Inenaga Y., Endo T. and Iwase T., "Automated detection method for architectural distortion with spiculation based on distribution assessment of mammary gland on mammogram", Digital Mammography / IWDM, Vol. 4046, pp. 370-375, 2006.

[11] Haralick R. M., "Statistical and structural approaches to texture", Proceedings of the IEEE, Vol. 67, No. 5, pp. 786-804, 1979.

[12] Ichikawa T., Matsubara T., Hara, H. Fujita T., Endo T. and Iwase T., "Automated detection method for architectural distortion areas on mammograms based on morphological processing and surface analysis", 
Proceedings of SPIE Medical Imaging, Image Processing, Vol. 5370, pp. 920-925, 2004.

[13] James Theiler, "Estimating Fractal Dimension", Journal of the Optical Society of America A, Vol. 7, No. 6, pp. 1055-1073, 1990.

[14] Karssemeijer N. and te Brake G. M., "Detection of stellate distortions in mammograms", IEEE Transactions on Medical Imaging, Vol. 15, No. 5, pp. 611-619, 1996.

[15] Kurt Hornik, "Multilayer feedforward networks are universal approximators", Neural Networks, Vol. 2, No. 5, pp. 359-366, 1989.

[16] Laws K. I., "Rapid texture identification", Proceedings of SPIE: Image Processing for Missile Guidance, Vol. 238, pp. 376-380, 1980.

[17] Magdalena Jasionowska, Artur Przelaskowski and Rafai Jozwiak, "Characteristics of Architectural Distortion in Mammograms - Extraction of Texture Orientation with Gabor filters", ICCVG 2010, Part 1, Vol. 6374, pp.420430, 2010.

[18] Matsubara T., Ichikawa T., Hara T., Fujita H., Kasai S., Endo T. and Iwase T., "Automated detection methods for architectural distortions around skinline and within mammary gland on mammograms", International Congress Series, International Congress and Exhibition on Computer Assisted Radiology and Surgery, Vol. 1256, pp. 950-955, 2003.

[19] Matsubara T., Hara T., Fujita H., Endo T. and Iwase T., “ Automated detection method for mammographic spiculated architectural distortion based on surface analysis", International Congress and Exhibition on Computer Assisted Radiology and Surgery (CARS2008), Vol. 3, No. 1, pp. S176-S177, 2008.

[20] Nakayama R., Watanabe R., Kawamura T., Takada T., Yamamoto K. and Takeda K., "Computer aided diagnosis scheme for detection of architectural distortion on mammograms using multiresolution analysis", International Congress and Exhibition on Computer Assisted Radiology and Surgery (CARS 2008), Vol. 3, No.1, pp. S418-S419, 2008.

[21] Nemoto M., Honmura S., Shimizu A., Furukawa D., Kobatake H. and Nawano S., "A pilot study of architectural distortion detection in mammograms based on characteristics of line shadows", International Journal of Computer Assisted Radiology and Surgery, Vol. 4, No.1, pp. 27-36, 2009.

[22] Özekes S., Osman O., and Çamurcu A.Y., "Computerized detection of architectural distortions in digital mammograms", International Conference on Computer Assisted Radiology and Surgery (CARS2005), Vol. 1281, pp. 1396, 2005.
[23] Pinaki Pratim Acharjya, Ritaban Das and Dibyendu Ghoshal, "A Study on Image Edge Detection Using the Gradients", International Journal of Scientific and Research Publications, Vol. 2, No. 12, pp. 1-5, 2012.

[24] Rangaraj M. Rangayyan, Jayasree Chakraborty, Shantanu Banik, Sudipta Mukhopadhyay and Leo Desautels J. E., "Detection of Architectural Distortion Using Coherence in Relation to the Expected Orientation of Breast Tissue", IEEE Transactions on CBMS, pp. 14, 2012.

[25] Shantanu Banik, Rangaraj M.Rangayyan and Leo Desautels J. E., "Detection of Architectural Distortion in Prior Mammograms", IEEE Transactions on Medical Imaging, Vol. 30, No. 2, pp. 279-294, 2011.

[26] Sujjoy Kumar Biswas and Dipti Prasad Mukherjee, "Recognizing Architectural Distortion in Mammogram: A Multiscale Texture Modeling Approach with GMM", IEEE Transactions on Biomedical Engineering, Vol. 58, No.7, pp. 2023-2030, 2011.

[27] Sampat M. P., Whitman G. J., Markey M. K. and Bovik A. C., "Evidence based detection of spiculated masses and architectural distortion", SPIE Medical Imaging 2005, Image Processing, Vol. 5747, pp. 26-37, 2005.

[28] Sampat M. P., Markey M. K. and Bovik A. C., "Measurement and detection of spiculated lesions", Image Analysis and Interpretation, pp. 105-109, 2006.

[29] Suckling J., Parker J., Dance D., Astley S., Hutt I., Boggis C., Ricketts I., Stamatakis E., N. Cerneaz, S. Kok, P. Taylor, D. Betal, and J. Savage, "The Mammographic Image Analysis Society Digital Mammogram Database", Experta Medica International Congress Series, Vol. 1069, pp. 375-378, 1994.

[30] Tourassi G. D., Delong D. M. and Floyd Jr C. E., “A study on the computerized fractal analysis of architectural distortion in screening mammograms", Physics in Medicine and Biology, Vol. 51, No. 5, pp. 1299-1312, 2006.

[31] Uhrig R.E., "Introduction to Neural Networks", IEEE Transactions on Industrial Electronics, Control and Instrumentation, Vol. 1, pp. 33-37, 1995.

[32] William T. Freeman and Edward H. Adelson, "The Design and Use of Steerable Filters", IEEE Transactions on Pattern Analysis and Machine Intelligence, Vol. 13, No. 9, pp. 891-906, 1991.

[33] Xinhua Zhuang., Haralick R.M. and Stanley Stenberg R., "Image Analysis using Mathematical Morphology", IEEE Transactions on Pattern Analysis and Machine Intelligence, Vol. 9, No. 4, pp. 532-550, 1987. 